\title{
Joint Stiffness Compensation for Application in the EXTEND Hand Orthosis
}

\author{
Claudia J.W. Haarman, Edsko E.G. Hekman, Gerdienke B. Prange and Herman van der Kooij
}

\begin{abstract}
This paper presents a passive hand orthosis, called EXTEND, that can be used during activities of daily living. In the design a negative stiffness spring mechanism is incorporated to overcome the high finger joint stiffness of stroke patients with hypertonia. The passive mechanism can be easily tuned by the user to compensate a linear joint stiffness of 0.15 to 0.33 $\mathrm{Nm} / \mathrm{rad}$ for each finger independently. A prototype was tested with four patients showing an increased functional ability of the hand during several tasks. With the orthosis, patients were better able to pick up mid-sized objects $(5-7.5 \mathrm{~cm}$ diameter) of different shapes.
\end{abstract}

\section{INTRODUCTION}

Hands allow humans to interact with their environment and perform activities of daily living (ADL). Stroke, or cerebrovascular accident (CVA), are among the primary causes of persisting disability [1]. Limited finger and wrist extension after stroke leads to loss of hand function. This is caused by hypertonicity or spasticity of the flexor muscles and/or weakness of the extensor muscles [2].

A hemiparesis is seen in more than $80 \%$ of the stroke survivors [3]. Despite intensive rehabilitation programs many patients continue to have problems that limit the functional capability of their hands and self-reliance. Only a small number of the stroke survivors shows complete functional recovery of the upper limb after 6 months [4]. 70\% of the patients who initially experience upper-extremity paresis have residual impairment [5], [6].

Permanent assistive devices, often referred to as orthoses or exoskeletons, can aid users by providing finger extension assistance. Restoring the functionality of their hands will enable patients to lead a more independent life. Also, studies have shown that learned nonuse might be stopped or prevented [7].

Bos et al. presented an extensive overview with active and passive dynamic hand orthoses [8]. Many of the active devices are targeted at rehabilitation, often leading to bulky and complex designs. Recent developments, such as the Exo-Glove [9], Soft Robotic Glove [10] and Soft Robotic Exomusculature Glove [11], [12] are examples of slim active

\footnotetext{
*This project has received funding from the European Unions Horizon 2020 research and innovation programme under grant agreement No. 688857 (SoftPro).

C.J.W. Haarman, E.E.G Hekman, G.B. Prange and H. van der Kooij are with the Department of Biomechanical Engineering, University of Twente, 7522 NB Enschede, The Netherlands C.j.w.haarmaneutwente.nl

C.J.W. Haarman is with Hankamp Rehab B.V., Enschede, The Netherlands.

G.B. Prange is with Roessingh Research and Development, Enschede, The Netherlands.
}

exoskeletons that can assist users with hand opening during ADL. However, passive and simple devices often lead to higher user acceptance rates as these are easier to design, control and maintain [13].

We aim to increase the hand function of stroke survivors with hypertonia through a passive hand orthosis. The design and testing of this new orthosis called EXTEND is discussed in this paper.

\section{BACKGROUND}

\section{A. Joint Stiffness}

The human finger has three joints: the metacarpophalangeal (MCP) joint, proximal interphalangeal (PIP) joint and distal interphalangeal (DIP) joint. Agonistic and antagonistic muscles control the rotations of these joints. A resistance to elongation is present, referred to as 'muscle tone'. Many stroke patients have increased muscle tone, called hypertonia [14]. Hypertonia results in an increased resistance against movement. Spasticity is a velocity-dependent increase in resistance. Hypertonia is not dependent on velocity and can be present with or without spasticity.

The muscle tone leads to passive joint stiffness, defined as the rotational stiffness that expresses the ratio of the moment to the rotation angle. Kamper et al. investigated the mechanical resistance to rotation of the MCP joint in stroke patients [15], [16]. They found that the torque required to extend hypertonic MCP joint is nearly linear with respect to the joint angle, thus showing an almost constant rotational stiffness. Brokaw et al. found a similar, approximately linear, relationship between the MCP torque and joint angle [17]. Finger joint stiffness values found in these studies varied between approximately 0.45 and $0.60 \mathrm{Nm} / \mathrm{rad}$.

\section{B. State of the Art}

Several passive devices use parallel springs on the dorsal side of the hand to assist hand opening by pulling on the fingers. These parallel springs cause a shift of the neutral point, i.e. the amount of hand opening in rest, but decrease the range of motion. Examples are the SaeboFlex [18], SaeboGlove (Saebo Inc; Charlotte, North Carolina) and SCRIPT [19].

The Arm Assist hand module [20] applies a near-constant opening force by elastic cords and a variable spring moment arm. Thus, the neutral point is shifted while the range of motion is preserved. All fingers are moved simultaneously.

The HandSOME [17] has a similar design. Its four-bar mechanism is mounted on the dorsal side of the hand and can follow the trajectory of the fingers. With this device 
A

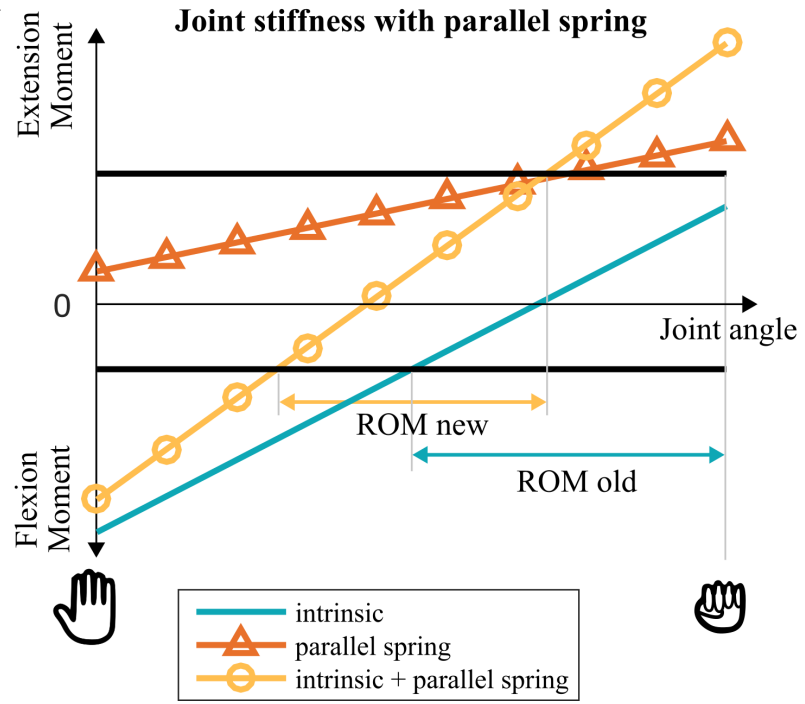

B

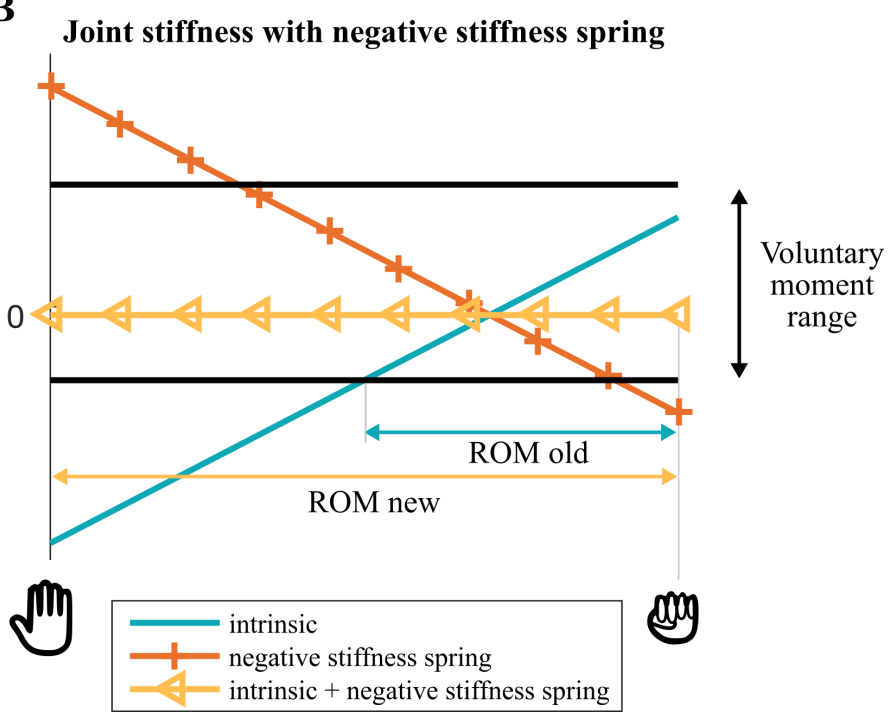

Fig. 1. Moment-angle graphs for different compensating strategies. (A) Moment due to intrinsic joint stiffness (blue) compensated with a parallel spring (orange). The resulting moment (yellow) leads to a shifted and decreased ROM. (B) Moment due to joint stiffness (blue) compensated with a negative stiffness spring (orange). The resulting moment (yellow) is zero. Therefore the maximum ROM is increased.

only gross movements are possible. The finger joints are not properly aligned with the rotational axes of the mechanism. Also, accounting for different levels of hypertonia throughout the day is not straightforward since only a discrete number of spring attachment points can be selected.

\section{DESIGN APPROACH}

\section{A. Extension Assistance}

In patients with moderate to severe joint stiffness the intrinsic flexion moment (resistance against extension) increases excessively with finger extension. This has a negative impact on the range of motion, which is bounded by the maximum voluntary flexion and extension moments. An example moment-angle profile of this intrinsic stiffness is plotted in Fig. 1.

Fig. 1A shows the situation when parallel springs are used to compensate for the intrinsic stiffness of the finger joints. Parallel springs (orange) add an increasing moment with flexion. The resulting moment-angle graph (yellow) has a larger positive slope than the intrinsic curve. This shifts and limits the range of motion.

Ideally, the added extension moment should exactly compensate the joint rotational stiffness. This requires a spring mechanism with negative stiffness. Negative stiffness springs have been used before in other applications to achieve an assistive moment. Early examples include a compensation of cosmetic glove stiffness for prosthetics and a flexible robot arm [21]-[23]. The Laevo exoskeleton applies a negative hip moment to compensate gravity torque during trunk flexion (Laevo B.V.; Delft, The Netherlands).

In our design we also applied a negative stiffness spring mechanism to increase the range of motion of the fingers. The effect can be seen in Fig. 1B. The patient can now easily move his or her fingers through the entire range of

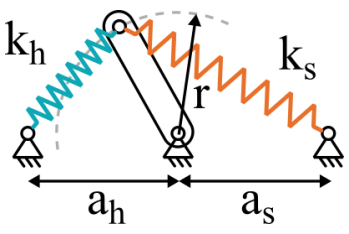

Fig. 2. Basic spring-to-spring balancer

motion because the resulting moment (yellow) stays within the voluntary moment range.

\section{B. Implementation of spring-to-spring balancing}

A basic spring-to-spring balancer is shown in Fig. 2 using zero-free-length springs. These springs have a force proportional to their length and are perfectly balanced if:

$$
a_{h} k_{h} r=a_{s} k_{s} r
$$

Where $k_{h}$ is the human finger joint stiffness, $k_{s}$ is the stiffness of the extension spring, $r$ is the length of the lever, and $a_{h}$ and $a_{s}$ are the distances of the pivot of the lever to the spring fixation points [24].

This principle is applied in the joint stiffness compensation mechanism of the hand orthosis (Fig. 3A). A torsion spring represents the human finger joint stiffness with stiffness $k_{h}$. The energy stored in a normal extension spring controls the amount of finger extension to cancel out the finger joint stiffness. The free length of this spring is stored behind a pulley to obtain a zero-free-length spring [24]. The extension spring applies a zero moment when the lever arm is in horizontal position. This corresponds to maximum finger joint flexion. When the finger extends the lever arm will rotate clockwise. Therefore the moment arm of the extension spring and thus the resulting extension moment increases, see also Fig. 3B). 
A
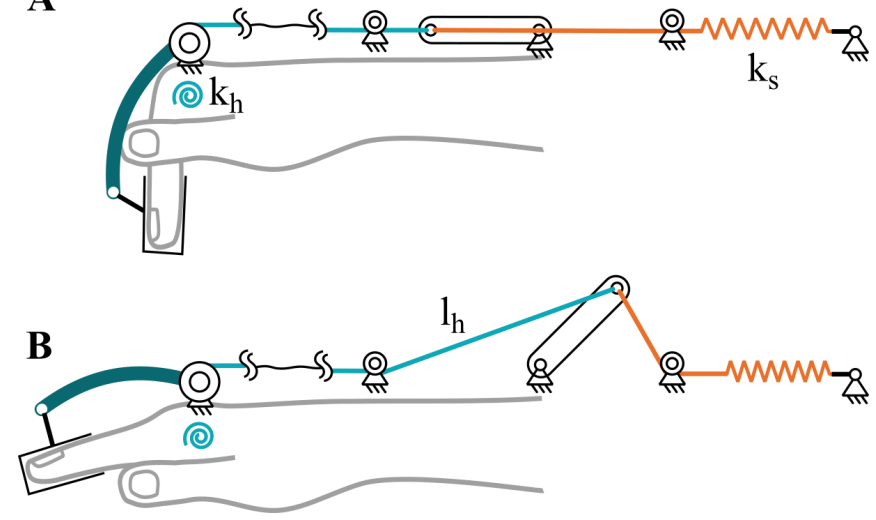

Fig. 3. (A) Spring-to-spring balancing mechanism applied to the finger in flexion. (B) Moment arm of extension spring increased when finger extends.

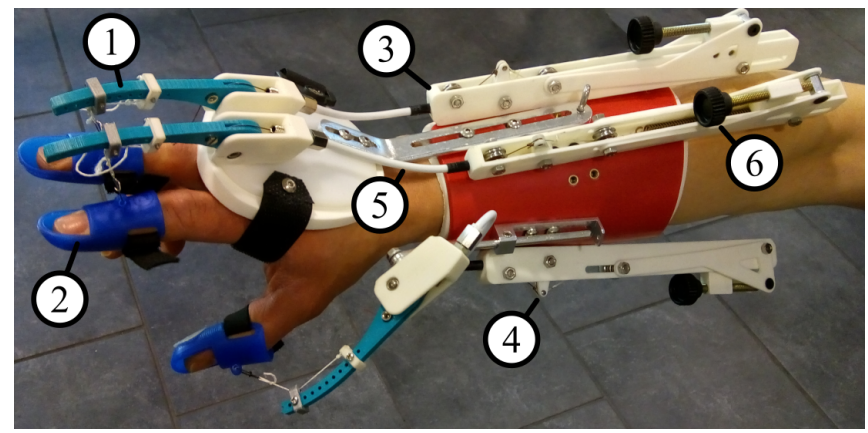

Fig. 4. Overview of the EXTEND prototype with the hand in extended position. (1) Lever arm; (2) Finger cap; (3) Stiffness compensation mechanism; (4) Lever arm negative stiffness mechanism; (5) Bowden cables; (6) Adjustment knob.

\section{Tuning the Assistance Profile}

Individual finger joint stiffness compensation adjustment allows the user to control the amount of extension assistance per finger. Increasing $a_{s}$ by a distance $\Delta x$ increases the finger joint stiffness $\left(k_{h}\right)$ that can be compensated.

The neutral (or zero-force) angle is the point where the moment due to joint stiffness is zero. Since the hypertonia causes the hand to be closed at rest, the neutral angle coincides with the closed hand configuration, which is maximum flexion. Changing cable length $\left(l_{h}\right)$, see Fig. 3B, allows adjustment of this point per finger.

\section{PROTOTYPE DESIGN}

The new device compensates the effects of flexor hypertonia to increase the functional ability of users. In Fig. 4 the EXTEND prototype is shown.

A lever arm (1) connects the finger cap (2) to a cable. The cable of each finger is guided to the stiffness compensation mechanism (3) placed above the arm cuff. The second lever arm (4) connects the cable to the negative stiffness spring. The wrist is fixed in a position that is comfortable for the patient. Bowden cables (5) transmit the forces from the mechanism to the fingers. In the prototype two fingers and the thumb are assisted and can be moved independently. The amount of assistance for each finger can be controlled

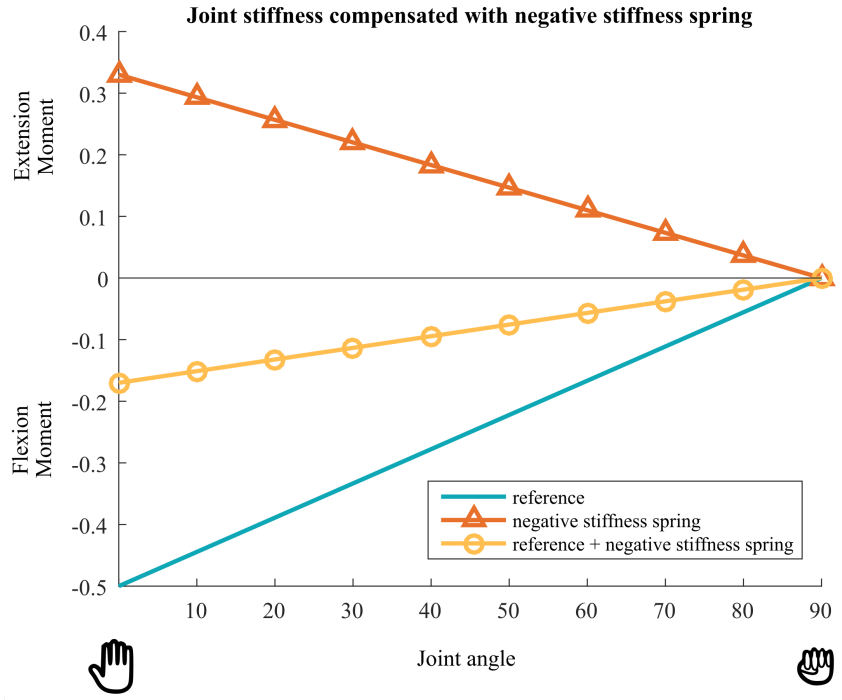

Fig. 5. Stiffness compensation with the negative stiffness mechanism Moment due to joint stiffness (blue) compensated with a negative stiffness spring (orange). The resulting moment (yellow) is decreased.

by rotating a knob that (6) can be easily reached by the unaffected hand.

An extension spring with a stiffness of $k_{s}=0.66 \mathrm{~N} / \mathrm{mm}$ is chosen. The value of $a_{s}$ can be manually adjusted from $a_{h}$ to $a_{h}+35 \mathrm{~mm}$. Length $a_{h}$ is set to $28 \mathrm{~mm}$. This will result in an effective spring stiffness range of:

$$
\begin{gathered}
k_{h \min }=\frac{a_{s} k_{s}}{a_{h}}=0.66 \mathrm{~N} / \mathrm{mm} \\
k_{\text {hmax }}=\frac{\left(a_{s}+35\right) k_{s}}{a_{h}}=1.48 \mathrm{~N} / \mathrm{mm}
\end{gathered}
$$

The lever arm above the MCP joint guides the cable along a radius $\left(r_{m c p}\right)$ of $15 \mathrm{~mm}$, resuling in a cable travel $(\mathrm{dx})$ of 15 $\mathrm{mm} / \mathrm{rad}$. As a result the forces in the cable $\left(F_{\text {cable }}=k_{h} d x\right)$ vary from 9.9 to $22.2 \mathrm{~N} / \mathrm{rad}$ in the minimum resp. maximum compensation setting.

A constant moment is applied to the lever arm:

$$
M_{\text {ext }}=F_{\text {cable }} \cdot r_{m c p}
$$

The selected extension spring $\left(k_{s}=0.66 \mathrm{~N} / \mathrm{mm}\right)$ can thus provide compensation of finger joint stiffnesses ranging between 0.15 to $0.33 \mathrm{Nm} / \mathrm{rad}$.

Fig. 5 shows the resulting torque-angle profile when a joint stiffness of $0.5 \mathrm{Nm} / \mathrm{rad}$ is compensated with the EXTEND orthosis. Assumed is a full range of motion from 0 to 90 degrees. From this figure it becomes clear that the new mechanism is able to reduce the required flexion torque to less than $0.2 \mathrm{Nm}$ at maximum finger extension.

\section{TESTING}

The functionality and usability of the prototype was tested with four patients suffering from hypertonia of the finger flexors. The purpose of the experiment was to compare 


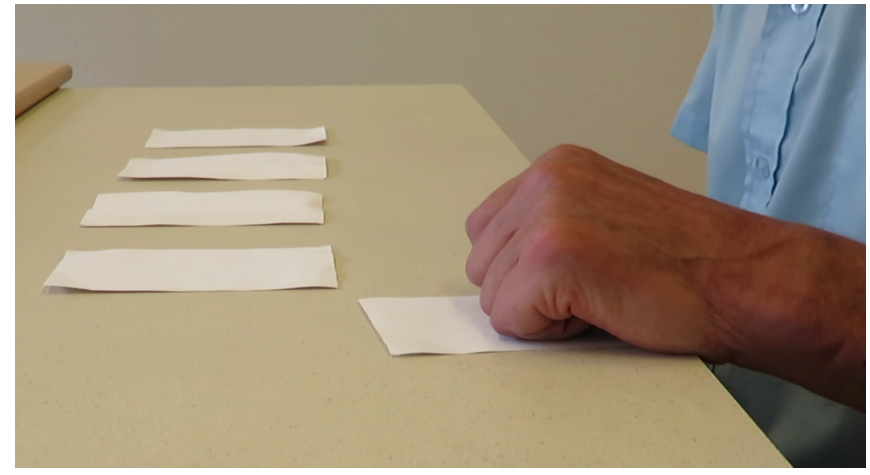

Fig. 6. Closed hand resting position of subject S3 without orthosis during the 'turning cards' task of the JTHFT.

the differences in hand functionality with and without the developed orthosis.

To determine how the orthosis can improve ADL tasks, several items of validated clinical ability tests that measure performance (time) and capacity (how well a task can be executed) are performed with and without orthosis and the differences are recorded. The Jebsen Taylor Hand Function Test (JTHFT) measures both time and quality of ADL tasks [25]. The test consists of 7 tasks, ranging from picking up small objects to moving empty and weighted large cans. The Action Research Arm Test (ARAT) focuses more on manual dexterity [26]. The arm function was not taken into account. Therefore subjects were instructed to support their affected arm with their unaffected hand during the tests.

Usability of the system was captured with an interview after the testing.

\section{A. Patient Recruitment}

Four stroke patients were recruited from the Roessingh Rehabilitation Center (Enschede, NL). Their characteristics can be found in Table I The patients were not limited in passive finger extension, but active extension was reduced due to hypertonia of the flexor muscles. They all had a closed hand in rest (Fig. 6). Patients with moderate to severe signs of spasticity in the fingers were excluded from the study. This was manually tested by passively extending the fingers of the patients and measuring the resistance at different speeds. All subjects gave their informed consent prior to the study.

\section{RESULTS}

Changes in functionality with and without orthosis were compared by performing several tasks of the ARAT and JTHFT. Also a short interview at the end of the test was conducted to obtain information about the usability of the design.

\section{A. ARAT}

Small improvements of hand functionality were seen with orthosis. Due to the limited arm function and almost absent finger movements without orthosis, adding the orthosis would increase the scores of (some of) the ARAT items from
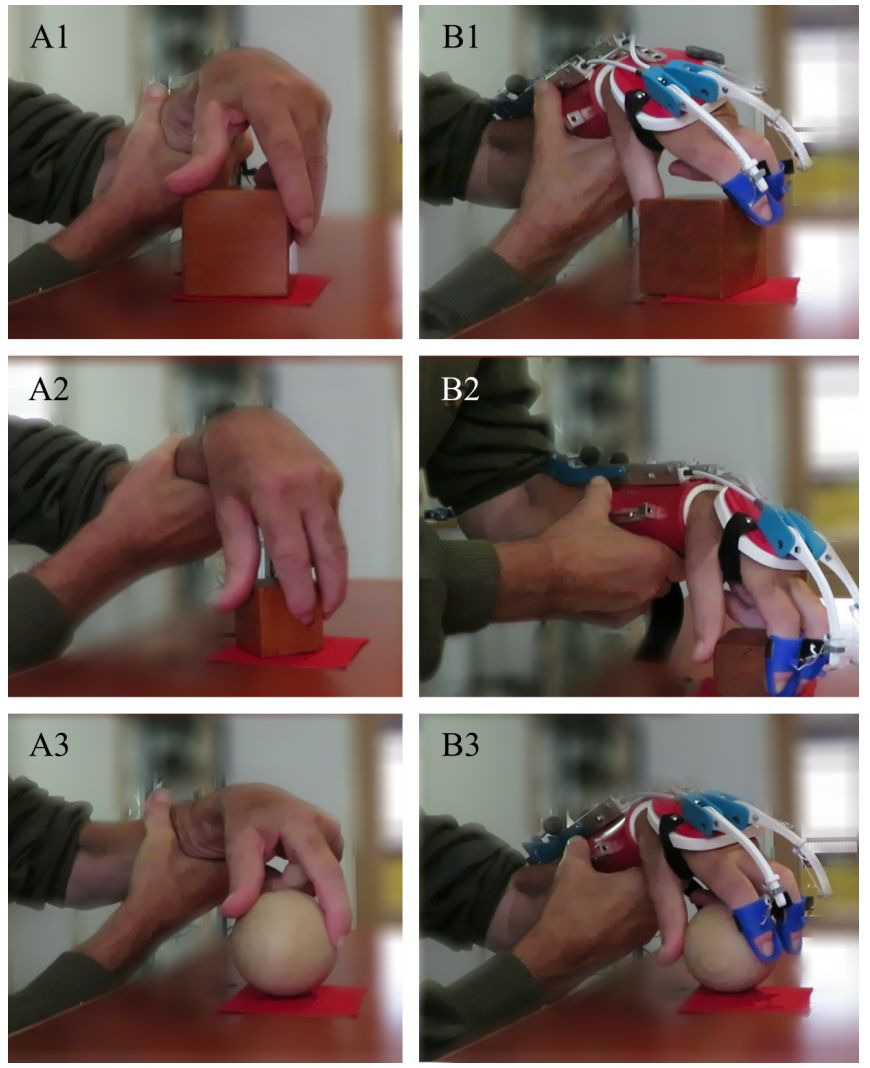

Fig. 7. Subject $\mathrm{S} 1$ showing unsuccessful completion of items the ARAT test without orthosis (A). With orthosis (B) the items were completed successfully. (1) block $7.5 \mathrm{~cm}$, (2) block $5 \mathrm{~cm}$, (3) ball

0 to 1 . In some cases the scores improved from 1 to 2 . In Table II the complete scores of all subjects can be found.

For subject S2 we saw a shift from the ability to pick up small items without orthosis to the ability to pick up large items. Subject S3 was unable to complete the test with orthosis.

In Fig. 7 the differences between picking up objects without (A) and with (B) orthosis can be seen for subject S1. These items of the ARAT are wooden blocks with a size of 7.5 (1) and $5 \mathrm{~cm}$ (2). The bottom image (3) shows a wooden ball. The orthosis did not improve the grasping of small objects $(<5 \mathrm{~cm})$ due to the lack of friction of the finger caps. This particular subject showed slight thumb extension with only the index and middle finger connected to the system. Since the finger cap degraded the functional capabilities of the thumb, it was decided to remove the thumb cap for this patient.

\section{B. JTHFT}

Small improvements were seen during the JTHFT as well. For a complete overview of the results, see Table II. For the JTHFT the maximum task time of $120 \mathrm{sec}$. was in most cases not enough to successfully complete the task. However, for most tasks the number of objects that could be grasped improved with orthosis. In Fig. 8 two items of the test are shown without (A) and with (B) orthosis for subject S1. The tasks are Picking up small objects (1) and Picking up 
TABLE I

CHARACTERISTICS OF TEST SUBJECTS

\begin{tabular}{|c||c||c||c||c||c|}
\hline Subject & Gender & Age(yr) & Time post-stroke (months) & Affected side & Dominant side (pre-stroke) \\
\hline S1 & $\mathrm{m}$ & 72 & 6 & Left & Right \\
\hline S2 & $\mathrm{m}$ & 66 & 8 & Right & Right \\
\hline S3 & $\mathrm{m}$ & 60 & 7 & Left & Right \\
\hline S4 & $\mathrm{m}$ & 65 & 47 & Left & Right \\
\hline
\end{tabular}

TABLE II

ARAT AND JEBSEN TAYLOR HAND FUnCTION TEST SCORES Without AND With THE EXTEND ORTHOSIS.

\begin{tabular}{|c||c||c||c||c|}
\hline Subject & ARAT scores without orthosis & ARAT scores with orthosis & JTHFT scores without orthosis & JTHFT scores with orthosis \\
\hline S1 & 5 & 8 & 106 & 96 \\
\hline S2 & 24 & 15 & 35 & 107 \\
\hline S3 & 6 & - & - & - \\
\hline S4 & 57 & 56 & 7 & 10 \\
\hline
\end{tabular}

weighted cans (2). Without orthosis this patient was not able to extend his index and middle finger to grasp the objects. With orthosis the patient was able to pick up the bottle cap and weighted can. The scores do not completely reflect the improvement. Subject S1 for example turned 5 instead of 3 cards. Also, he was able to pick up 5 small common objects instead of 0, and picked up 4 weighted cans with the orthosis. Subject S2 had some issues picking up small objects due to lack of grip of the finger caps. This heavily affected the scores of this test. Subject S3 was too tired to successfully complete the JTHFT. Subject S4 who had a relatively good hand function, did not experience much hinder of the orthosis during execution of the test items.

\section{Usability}

Regarding usability of the system, subjects valued the adjustability of the amount of assistance, independent movement of fingers and nice distribution of force during motion. Also, they thought the system was easy to understand. Three subjects noticed that the finger caps reduce touch sensation of fingertips during object manipulation. This makes it difficult to grasp small objects. Two subjects indicated they would like to control all five fingers, instead of three fingers if grip issues with the finger caps are solved.

One subject did not use the negative stiffness mechanism to actively move the thumb. However when moving his other finger, spontaneous movement of the thumb was seen. The hand-mounted parts together weigh $110 \mathrm{~g}$. which was considered light enough for daily use. However, the arm cuff including three finger stiffness compensation mechanisms weighs $490 \mathrm{~g}$. which is regarded too heavy by three patients. All subjects were positive about the device and would like to use the orthosis in a home setting when the most important disadvantages (size, weight, lack of touch sensation) are removed.

\section{CONCLUSIONS AND DISCUSSION}

The developed orthosis for ADL contains a negative stiffness spring system to compensate the increased finger stiffness of stroke patients. The presented design supports the affected hand during bimanual tasks. These are primarily stabilizing tasks such as pouring liquids into a glass, and holding small and large objects of different shapes. The passive mechanism can be tuned by the user to compensate joint stiffness in the range of 0.15 to $0.33 \mathrm{Nm} / \mathrm{rad}$. This is considered sufficient to drastically decrease the amount of muscle force needed to move the hand. In a next iteration of the design the amount of joint stiffness that can be compensated should be increased.

Experimental results from functional tests such as the ARAT and JTHFT showed in some cases improvements in hand function. Although the improvements reflected by the ARAT and JTHFT scores may not seem large, for the patient they mean a difference from complete dependence to be able to live a more independent life. The small or absent increases in hand function were mainly attributed to the slippery and bulky finger caps which is considered a major improvement point. Also, the use of different caps with increased friction and soft interface can increase touch sensation and improve donning.

The mechanism is moved away from the hand towards the forearm to lower the weight that is applied to the hand. However, the weight and size of the arm-mounted part were still considered to be too high. Due to limited arm function, the subjects were not able to lift their affected arm without help of their unaffected hand. Adding a relatively large weight of $600 \mathrm{gr}$. further enhances this effect. A new design should have a reduced weight of maximum $300 \mathrm{gr}$.

The moment applied by the mechanism to the MCP joint was assumed linear. However, due to geometric constraints, the link that attaches the cable to the fingertip is mounted above the MCP joint rotation axis. The moment arm is therefore not constant, limiting the linearity of the system. Since reference data of finger joint stiffness of stroke patients with hypertonia was not readily available, further research will include measurements of the torque required to passively extend the finger. With this information the assumption of a linear joint stiffness can be verified. Also, the stiffness properties of the thumb will be investigated, since no reference 

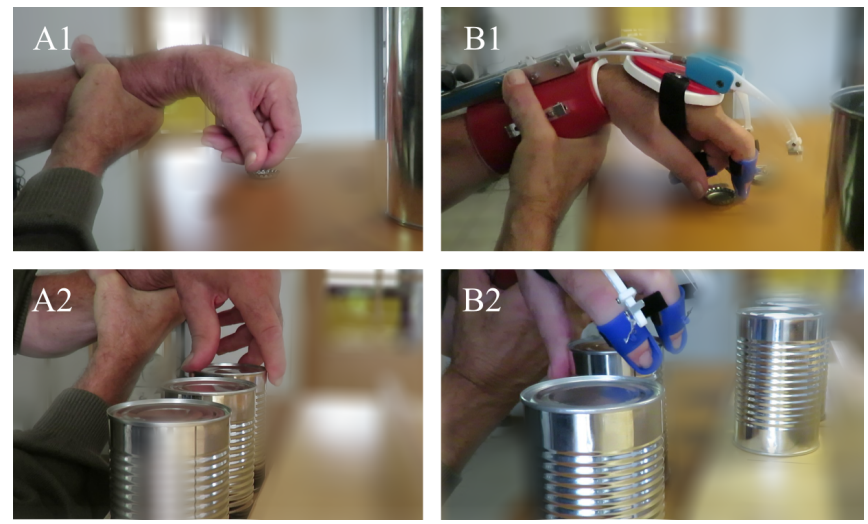

Fig. 8. Subject S1 showing unsuccessful completion of items of the JTHFT without orthosis (A). With orthosis (B) the items were completed successfully. (1) Picking up small objects. (2) Picking up weighted cans.

data could be found in literature.

Future research will furthermore involve measurements of the grip force and ROM data from the unsupported hand, the hand with negative stiffness mechanism, and the hand with a conventional orthosis containing parallel springs to allow for a direct comparison between these situations.

The principle of negative stiffness compensation as displayed in this research has the potential to be used in passive devices but also in actively controlled hand exoskeletons. Since already a large portion of the desired torque will be provided by the passive spring system, the required output force of the motor can be lowered. These relatively lightweight and small motors can now take over the fine adjustment, accounting for varying finger joint stiffness throughout the day. The next generation of mechatronic hand systems can have added functionality without the need for high-torque motors to compensate the hypertonia of the patient.

\section{REFERENCES}

[1] Writing Group Members, "Heart Disease and Stroke Statistics2014 Update: A Report From the American Heart Association," Circulation, vol. 129, no. 3, pp. e28-e292, jan 2014. [Online]. Available: http://www.ncbi.nlm.nih.gov/pmc/articles/PMC5408159/

[2] P. N. O'Dwyer NJ, L. Ada, "Spasticity and muscle contracture following a stroke," Brain, vol. 119, no. February, pp. 1737-49, 1996.

[3] H. Nakayama, H. S. Jorgensen, H. O. Raaschou, and T. S. Olsen, "Compensation in recovery of upper extremity function after stroke: the Copenhagen Stroke Study." Archives of physical medicine and rehabilitation, vol. 75, no. 8, pp. 852-857, aug 1994.

[4] G. Kwakkel, B. J. Kollen, J. V. Van der Grond, and A. J. H. Prevo, "Probability of regaining dexterity in the flaccid upper limb: Impact of severity of paresis and time since onset in acute stroke," Stroke, vol. 34, no. 9, pp. 2181-2186, 2003.

[5] D. T. Wade, "Measuring arm impairment and disability after stroke." International disability studies, vol. 11, no. 2, pp. 89-92, 1989.

[6] L. Richards and P. Pohl, "Therapeutic interventions to improve upper extremity recovery and function," Clinics in geriatric medicine, vol. 15 , no. 4, pp. 819-832, 1999.

[7] E. Taub, G. Uswatte, V. W. Mark, and D. M. M. Morris, "The learned nonuse phenomenon: implications for rehabilitation." Europa medicophysica, vol. 42, no. 3, pp. 241-56, 2006.

[8] R. A. Bos, C. J. W. Haarman, T. Stortelder, K. Nizamis, J. L. Herder, A. H. A. Stienen, and D. H. Plettenburg, "A structured overview of trends and technologies used in dynamic hand orthoses," Journal of
NeuroEngineering and Rehabilitation, vol. 13, no. 1, p. 62, 2016 [Online]. Available: http://dx.doi.org/10.1186/s12984-016-0168-z

[9] H. In, B. B. Kang, M. Sin, and K. J. Cho, "Exo-Glove: A Wearable Robot for the Hand with a Soft Tendon Routing System," IEEE Robotics \& Automation Magazine, vol. 22, no. 1, pp. 97-105, 2015.

[10] H. K. Yap, J. H. Lim, J. C. H. Goh, and C.-H. Yeow, "Design of a Soft Robotic Glove for Hand Rehabilitation of Stroke Patients With Clenched Fist Deformity Using Inflatable Plastic Actuators," Journal of Medical Devices, vol. 10, no. 4, p. 044504, 2016.

[11] M. A. Delph, S. A. Fischer, P. W. Gauthier, C. H. M. Luna, E. A. Clancy, and G. S. Fischer, "A soft robotic exomusculature glove with integrated sEMG sensing for hand rehabilitation," in 2013 IEEE 13th International Conference on Rehabilitation Robotics (ICORR), 2013, pp. 1-7.

[12] C. J. Nycz, M. A. Delph, and G. S. Fischer, "Modeling and design of a tendon actuated soft robotic exoskeleton for hemiparetic upper limb rehabilitation," Proceedings of the Annual International Conference of the IEEE Engineering in Medicine and Biology Society, EMBS, pp. 3889-3892, 2015.

[13] S. Balasubramanian, J. Klein, and E. Burdet, "Robot-assisted rehabilitation of hand function." Current opinion in neurology, vol. 23, no. 6, pp. 661-70, 2010.

[14] C. Marciniak, "Poststroke Hypertonicity: Upper Limb Assessment and Treatment," Topics in Stroke Rehabilitation, vol. 18, no. 3, pp. 179194, may 2011.

[15] D. G. Kamper and W. Z. Rymer, "Quantitative features of the stretch response of extrinsic finger muscles in hemiparetic stroke," Muscle and Nerve, vol. 23, no. 6, pp. 954-961, 2000.

[16] D. G. Kamper, R. L. Harvey, S. Suresh, and W. Z. Rymer, "Relative contributions of neural mechanisms versus muscle mechanics in promoting finger extension deficits following stroke," Muscle and Nerve, vol. 28, no. 3, pp. 309-318, 2003.

[17] E. B. Brokaw, I. Black, R. J. Holley, and P. S. Lum, "Hand Spring Operated Movement Enhancer (HandSOME): A portable, passive hand Exoskeleton for stroke rehabilitation," IEEE Transactions on Neural Systems and Rehabilitation Engineering, vol. 19, no. 4, pp. 391-399, 2011.

[18] J. F. Farrell, H. B. Hoffman, J. L. Snyder, C. A. Giuliani, and R. W. Bohannon, "Orthotic aided training of the paretic upper limb in chronic stroke: results of a phase 1 trial." NeuroRehabilitation, vol. 22, no. 2, pp. 99-103, 2007.

[19] S. Ates, C. J. W. Haarman, and A. H. A. Stienen, "SCRIPT passive orthosis: design of interactive hand and wrist exoskeleton for rehabilitation at home after stroke," Autonomous Robots, vol. 41, no. 3, pp. 711-723, 2017. [Online]. Available: https://doi.org/10.1007/s10514-016-9589-6

[20] N. R. Butler, S. A. Goodwin, and J. C. Perry, "Design parameters and torque profile modification of a spring-assisted hand-opening exoskeleton module," in IEEE International Conference on Rehabilitation Robotics, 2017, pp. 591-596

[21] J. Kruit, "Otto Bock gloves size 42 en 43, measurement thumb pivot, glove modification (In Dutch)," WMR, no. Delft university of Technology, Delft., 1987.

[22] S. Hirose, "Biologically inpired robots," no. Oxford University Press, 1993.

[23] F. van den Berg, "Design of low-friction laparoscopic forceps in $5 \mathrm{~mm}$ version (in Dutch)," MSc-Thesis, vol. A-864, no. OCP-MMS, Delft University of Technology, 1999.

[24] J. Herder, Energy-free systems: theory, conception, and design of statically balanced spring mechanisms, 2001, no. November. [Online]. Available: http://repository.tudelft.nl/view/ir/uuid:8c4240fb0315-462a-8b3b-efbd0f0e68b6/

[25] R. H. Jebsen, N. Taylor, R. B. Trieschmann, M. J. Trotter, and L. A. Howard, "An objective and standardized test of hand function." Archives of physical medicine and rehabilitation, vol. 50, no. 6, pp. 311-319, jun 1969.

[26] R. C. Lyle, "A performance test for assessment of upper limb function in physical rehabilitation treatment and research." International journal of rehabilitation research, vol. 4, no. 4, pp. 483-492, 1981. 